\title{
Acute Metabolic Acidosis in an Adolescent with Anorexia Nervosa: a Transdisciplinary Approach
}

\author{
Nour El Ayoun Ibrahim ${ }^{1}$, Lissa-Claudia Yajima-Dupuis ${ }^{1}$, Tristan Morichau-Beauchant ${ }^{2}$, Béatrice Gal ${ }^{1}$, \\ Marie-Rose Moro ${ }^{1}$, *Corinne Blanchet-Collet ${ }^{1}$ \\ ${ }^{1}$ Maison de Solenn - Maison des Adolescents- Hôpital Cochin, AP-HP, Université Paris Descartes, France. \\ ${ }^{2}$ Service de Réanimation médicale - Hôpital Cochin, AP-HP, Université Paris Descartes, France.
}

Received: August 10, 2016; Accepted: August 25, 2016; Published: September 14, 2016

*Corresponding author: Corinne Blanchet-Collet, Maison de Solenn - Maison des Adolescents- 97 boulevard de Port Royal, Hôpital Cochin, APHP, CESP, INSERM, Univ. Paris-Descartes, USPC, Paris, France, Tel: +33-(0)1 584128 14 ; Fax: +33-(0)1 584128 09;

E-mail: corinne.blanchet@aphp.fr

Keywords: Anorexia Nervosa; Metabolic Acidosis; Bipolar Disorder; Transdisciplinarity

\section{Introduction}

Eating disorders are complex and multifactorial diseases with frequent metabolic complications in which the electrolytes disturbances may be life-threatening. These abnormalities are more often observed in patients with a binge/purging type of anorexia nervosa or with bulimia nervosa, which involves purging behavior and medications intake [1]. Beyond some cases of individual susceptibility, patients with restrictive anorexia nervosa generally do not tolerate massive and rapid dietary restriction. Accordingly, total aphagia, involving complete refusal of all food and liquid intake, is an indicator of severity that justifies admission in a specialized department for nutritional rescue. Similarly, ketonuria is an admission criterion according to the French Authority for Health (HAS) [2].

Ketonuria is thus the first metabolic repercussion of aphagia in anorexia nervosa physicians usually look for. Signs of poor tolerance appear next and may announce multiorgan decompensation; its initial signs are hepatic cytolysis, functional kidney failure, and hematologic changes such as severe neutropenia, which generally occurs before damage to other cell lines. We present a case of acute metabolic acidosis in an adolescent girl with nearly total aphagia due to mixed anorexia nervosa. We will examine the conditions that promoted the appearance of acidosis, a rare event in eating disorders, and look at the predictive psychosomatic factors involved.

\section{Case report}

A 15-years-old girl with a binge type of anorexia nervosa (alternation of restrictive and binge eating episodes) since the age of 13, was admitted to our department. Her eating disorder was associated with a DSM-5 medication-induced bipolar disorder (shift into a manic or hypomanic state under antidepressant treatment). The patient's mother had a history of type II bipolar disorder and committed suicide when her daughter was 6 years old. The father works, as did the mother, in the field of health care. The neonatal patient's history included cerebral palsy resulting in right hemidystonia. She was in ninth grade at school and had a certain level of dyspraxia (visiospatial and fine motor dyspraxia and syndrome, but no mental retardation) remarkably compensated by her hyperintense dedication at school.

Her maximum pre-morbid weight before the onset of anorexia nervosa was $52 \mathrm{~kg} / 150 \mathrm{~cm}$ (BMI: 23.1), and her minimum weight was $36 \mathrm{~kg} / 1 \mathrm{~m} 54$ (BMI: 15.2). Six months before this admission, she gained weight after beginning a neuroleptic treatment during a major binge-eating period; her weight reached $63 \mathrm{~kg}$ / 1m54 (BMI: 26.6). After this excessive weight gain, which she found very hard to accommodate on the psychological level, she quickly and voluntarily resorted to a massive reduction of her food intake, as a result of which she lost $28 \mathrm{~kg}$ in 5 months. She also spontaneously stopped the psychotropic treatment, which she blamed for the weight gain. Her psychological situation also deteriorated progressively, culminating in both total exhaustion and a resurgence of anorexic cognitions. This scheduled hospitalization was planned to reassess and adapt her treatment. At admission, we were struck by her cachexia, pallor, impaired general condition, and major physical and psychical lethargy, without mental confusion. She reported that she had gastrointestinal disorders for several days, specifically, nausea, without vomiting, and imprecise abdominal pain. However, she did not show any worry about her condition, but showed rather jubilation and an attitude of omnipotence about this extreme malnutrition. Her thoughts appeared automated and reduced to anorexic cognitions (body shape disturbance, fat phobia). There was neither conventional mood symptom nor suicidal ideation. Her family reported no recent general weakness but did describe physical hyperactivity, major sleep disorders, and a nearly total 
lack of food intake over the previous 10 days. Her intake during the previous 3 days appeared difficult to quantify and may have been limited to 100 or $200 \mathrm{ml}$ of orange juice per $24 \mathrm{~h}$. On clinical examination, her blood pressure was $98 / 54 \mathrm{~mm} \mathrm{Hg}$, her heart rate $101 \mathrm{bpm}$, her temperature $36.5^{\circ} \mathrm{C}$, and her Sp02 99\%. She weighed $35 \mathrm{~kg} / 154 \mathrm{~cm}$, with a BMI of 14.8 . The urinary dipstick measured ketones at four crosses without any glycosuria. The blood test at admission (D0) found a low level of total $\mathrm{CO}_{2}$ at 9 mmol/l. Blood gases showed compensated metabolic acidosis: $\mathrm{pH}=7.38, \mathrm{pCO}_{2}=23.6 \mathrm{mmHg}, \mathrm{pO}_{2}=11 \mathrm{~mm} \mathrm{Hg}, \mathrm{HCO}_{3}^{-}=14.4$ $\mathrm{mmol} / \mathrm{l}$. The anion gap was elevated at $20 \mathrm{mEq} / \mathrm{L}$. Lactate was normal. Others findings, except elevated liver enzymes, including the blood electrolyte analysis, blood glucose, and renal function, were unremarkable (Table1). Urinary toxins were negative. The ECG showed sinus tachycardia without either conduction disorders or repolarization, with a QTc of $450 \mathrm{~ms}$. The patient was then transferred to the intensive care unit of our hospital for management of compensated metabolic acidosis and for monitoring of her ventilation. Initial management involved intravenous (IV) hydration and electrolyte replacement with a G5\% solution $(1000 \mathrm{ml} / 24 \mathrm{~h})$ containing $8 \mathrm{~g}$ of $\mathrm{NaCl} / \mathrm{l}$ and 3 $\mathrm{g}$ of $\mathrm{KCl} / \mathrm{l}$ associated with phosphate, multivitamin, and oligo elements supplementation to prevent refeeding syndrome. At the same time, a progressive feeding tube enteral nutrition at 10 $\mathrm{Kcal} / \mathrm{Kg} / \mathrm{d}$ began. As the patient's blood sugar levels were normal, no insulin was administered parenterally. No bicarbonates were administered as the metabolic acidosis was due to ketoacids with an elevated anion gap.

\section{Clinical course}

The clinical course at D1 was positive, and the patient was

Table 1: Biological characteristics at admission (D0) and at the first (D1) and third days (D3) of hospitalization.

\begin{tabular}{|l|l|l|l|}
\hline & D0 & D1 & D3 \\
\hline $\begin{array}{l}\text { Blood glucose (N: 3.5-5.5 } \\
\text { mmol/L) }\end{array}$ & $3.5 \mathrm{mmol} / \mathrm{l}$ & $\mathrm{NR}$ & $4.1 \mathrm{mmol} / \mathrm{l}$ \\
\hline $\mathrm{Na}(\mathrm{N}: 136-146 \mathrm{mmol} / \mathrm{L})$ & 138 & 144 & 144 \\
\hline $\mathrm{K}(\mathrm{N}: 3.5-4.5 \mathrm{mmol} / \mathrm{L})$ & 4.4 & 3.5 & 3.1 \\
\hline $\begin{array}{l}\mathrm{CO}_{2} \text { total }(\mathrm{N}: 22-29 \\
\text { mmol/L) }\end{array}$ & $\mathbf{9}$ & 21 & 26 \\
\hline Ketonuria & ++++ & +++ & $\mathrm{N}$ \\
\hline Glycosuria & $\mathrm{N}$ & $\mathrm{N}$ & $\mathrm{N}$ \\
\hline Proteins (N: 65-81 g/L) & 70 & 55 & 58 \\
\hline $\begin{array}{l}\text { Creatinine (N: } 45-100 \\
\mu m o l / L)\end{array}$ & 55 & 47 & 46 \\
\hline Urea (N: $2.5-7.5 \mathrm{mmol} / \mathrm{L})$ & 2.0 & 1.6 & 1.7 \\
\hline ASAT (N: $10-45 \mathrm{IU} / \mathrm{L})$ & 26 & $\mathrm{NR}$ & 22 \\
\hline ALAT (N: $10-35 \mathrm{IU} / \mathrm{L})$ & $\mathbf{2 5 3}$ & $\mathrm{NR}$ & 22 \\
\hline $\begin{array}{l}\text { Phosphorus (N: } 0.8-1.4 \\
\text { mmol/L) }\end{array}$ & 1.29 & 1.4 & 1.56 \\
\hline Blog & & & \\
\hline
\end{tabular}

Blood tests showed a metabolic acidosis and hepatic cytolysis with elevated transaminase (ALAT) levels at seven times the upper value resulting from a severe starvation. Normalization of these values were observed after three days of progressive refeeding

(NR: not reported; N: negative). transferred back to our department. The course of the laboratory results was also satisfactory, with the alkaline reserve reaching $21 \mathrm{mmol} / \mathrm{l}$ at D1. Ketonuria nonetheless remained high at 3 crosses on D1, while serum phosphorus remained stable. Vitamin K1 was administered preventively in view of the slightly elevated ACT (activated clotting time) (42/33). Ketonuria returned to normal on D3 (Table 1).

Exclusively enteral nutrition $(750 \mathrm{kcal} / \mathrm{d})$ continued for one week, and then spontaneous refeeding was carefully reintroduced, progressively replacing the enteral nutrition. After a period of nocturnal enteral nutrition, definitive weaning from enteral nutrition took place at seven weeks of hospitalization. She reached a weight of $42 \mathrm{Kg} / 154 \mathrm{~cm}$ (BMI: 17.71). We observed no signs of refeeding syndrome. Psychological functioning changed considerably at the same time as weight gain began. In spontaneous discourse, anorexic cognitions were rapidly replaced by depressive thoughts: affective anesthesia, experience/perception of depersonalization, affective regression associated with early morning awakening. We rapidly reached a diagnosis of underlying mixed mood disorders in this patient with some degree of tachypsychia, an absence of fatigue, and invasive thoughts of death. The physical hyperactivity with morning paroxysm filled a dual function along the border of mood and eating disorders. That is, hyperactivity, used for self-destructive purposes, appears as an equivalent of suicide. At the same time, the patient was battling against emotional anesthesia by a frantic search for sensations. Five weeks after hospitalization (BMI: 17), the mixture of moods grew richer: melancoly statements, suicidal ideation, and incurability were associated with psychological excitation and transient incidents of euphoria. From that point in the disease, physical hyperactivity had an anxiolytic function. Somatic improvement made it possible to begin medication to regulate mood, which was stabilizing. The eating behaviors also became more stable. The episode of severe malnutrition was analyzed and maintains a traumatic value today.

\section{Discussion}

To our knowledge, this is the first case of metabolic acidosis connected to anorexia nervosa in adolescence reported in the literature. The physiological or pathological situations leading to the onset of ketosis are not rare in daily clinical practice. Very schematically, ketosis is induced by a low-carbohydrate diet or an insulin deficiency, leading to a reduction in the insulin/ glucagon ratio, which in turn results in the secretion by the liver of ketone bodies such as $\beta$-hydroxybutyric acid, acetic acid, and acetone. Instead of glucose they are used as energy substrate for the principal organs, especially the central nervous system. Ketone bodies are eliminated by the urinary and/or respiratory routes.

Beyond voluntary fasting situations, such as hunger strikes $[3,4]$, numerous cases of ketosis occurring in physiological or pathological situations have been described and provide some explanation of the pathophysiologic mechanisms of our ketoacidosis case reported. In type 1 diabetes, a more or less profound insulin deficiency can lead to ketoacidosis of variable 
severity. During pregnancy, physiological metabolic acidosis can occur after a short period of fasting $[5,6]$. In the second trimester, the secretion of placental hormones (human placental lactogen and progesterone) and maternal counter regulatory hormones (cortisol, glucagon, and growth hormones) induce a transient state of insulin resistance. Metabolic acidosis can also surprise clinicians by speed of occurrence when patients with chronic (active or detoxified) alcoholism are fasting [7]. Ethanol metabolism produces ketone bodies in these patients, who also have depleted glycogen levels. Similar complications have also been described in cases of bariatric surgery. Bruegger and Rehm reported the case of a young woman with a gastric band who complained of uncontrollable vomiting for a week. Radiography of the thorax showed that a lung disease was the cause of her vomiting and that it in turn led to metabolic acidosis [8]. Patients following a low-carbohydrate diet such as the Atkins diet, can also develop this type of metabolic complication $[9,10]$. These diets, which are low in carbohydrates and high in lipids or proteins, are called ketogenic diets and have the effect of maintaining a low insulin level and a high glucagon level. This induces the production of ketone bodies in three weeks. Finally, Mittal and Khan reported a case that appears closer to ours [11]. It involved a woman with no psychiatric history who presented with acute psychosis and urinary ketosis secondary to a prolonged lowcalorie diet. Examinations revealed a stroke in a woman with a factor $\mathrm{V}$ Leiden mutation. The stroke was clearly promoted by the dehydration induced by the severe dietary restriction and by the blood hyper viscosity induced by the factor $\mathrm{V}$ mutation. Our patient presented a binge type of anorexia nervosa, without purgative behaviors, with aphagia developing over an 8-day period following a voluntary regimen with progressive reactivation of anorexic mechanisms

We hypothesize that this metabolic acidosis was due to prolonged fasting: her carbohydrate intake had been nearly nonexistent for 8 days, the urinary dipstick showed ketone bodies, and the blood gases compensated metabolic acidosis. There was no evidence of any differential diagnosis. Questioning of the patient and family did not suggest any drug use or toxic ingestion. Urinary toxins were negative. Her glycemic level was normal. The urinary dipstick showed no glycosuria. Renal function was normal. Finally, the rapidly favorable course as early as D3, induced by exclusive enteral refeeding alone as the essential treatment, is strong evidence that confirmed this hypothesis. We did not use exogenous carbohydrates, bicarbonates, or insulin.

We propose the following pathophysiologic explanation. During a period of continuous fasting, reduced blood glucose reduces the secretion of insulin, which in turn causes lipase activity to rise, releasing free fatty acids. These are transformed into acetyl-coenzyme A, an intermediary in the Krebs cycle. However, during fasting, hepatic neoglucogenesis causes the Krebs cycle to stop. Accordingly, acetyl-coenzyme A is transformed into ketone bodies in liver cells and thus, enables energy production to continue in the absence of glucose. The acid overload causes the bicarbonates to combine with $\mathrm{H}+$ ions to generate $\mathrm{CO}_{2}$ and $\mathrm{H}_{2} \mathrm{O}$. The $\mathrm{CO}_{2}$ produced is then eliminated by the pulmonary route; this results in an isolated decrease in peripheral blood bicarbonates. If respiratory compensation is adequate, the $\mathrm{PaCO}_{2}$ drops proportionally with the reduction in the bicarbonate concentration. Compensatory hyperventilation may cause exhaustion of the respiratory muscles [12]. The $\mathrm{pH}$ level in these situations is rarely less than 7.3 because of residual insulin secretion [7].

We were particularly surprised by the severity of the clinical presentation. The long duration of the aphagia, the speed of the weight loss, and the physical hyperactivity probably promoted this acute metabolic decompensation.

We also hypothesize that our patient's phenotypic particularities and multifactorial vulnerability were associated with the severity of the situation. In addition to familial and individual psychiatric comorbidities, the neonatal neurological history, has probably worsened the distortion of body image, and the lability of the eating disorders symptoms.

Considering the patient's premorbid condition, we note that several aspects of her familial history might well have contributed to the localization in the body of this unusual and extreme psychological disorder. Both the adolescent's construction of her identity and intrafamily communication were organized around her disabilities and somatic disorders. The process of identification has developed around a representation of the mother simultaneously physically ill (neoplasia) and selfdestructive (multiple addictions, suicide). Inhibition of thought and major alexithymia of multifactorial origin (lack of a parental reflective function, defenses against her mother's traumatic death in childhood, and repeated teasing by peers) make recourse to action particularly necessary. When we focus on the psychiatric situation, it appears that her mixed mood condition, revealed by the cognitive restoration may have contributed to the acute aphagia. In terms of psychopathology, the following description may explain the situation: a depressed patient seeks not only to surrender to death but to kill herself; refusing food, which can range from a lack of appetite to active resistance, is one expression of this aim. Exaltation, typical of the anorexic in her challenge to death, is maintained by a feeling of omnipotence characteristic of the manic dimension. The chronology of the symptoms shows us the reciprocal influence of anorexic cognitions and behavior on the mood disorders: a function of the fight against depression at the initial phase, then a factor in strengthening the depression by the malnutrition it induces. Moreover we see that the physical hyperactivity cannot be reduced to behavior that compensates for food intake. In their literature review of the comorbidity between bipolar and eating disorders, Mc Elroy et al [13, 14] found a higher prevalence of eating disorders in patients with bipolar disorders (2.3 to 15\% compared with $5 \%$ in the general population) as well as a higher prevalence of bipolar disorders in patients with an eating disorder (1.6 to 63\% compared with $10 \%$ in the general population). The most frequent comorbidity, according to the authors, concerned bulimia and type II bipolar disorder (depressive episodes alternating with hypomanic states). Similarities between eating and bipolar disorders 
are found for their phenomenology, progress, family history, laboratory findings, and treatment.

Finally, the rarity of metabolic acidosis in anorexia nervosa would appear to be directly correlated to the young woman's phenotypic complexity and to the multiplicity of existing vulnerability factors, such as we presented here.

\section{Conclusion}

The onset of a metabolic acidosis in a patient with anorexia nervosa remains very rare, but also exceptionally severe and unknown to physicians. To our knowledge, this is the first reported case of starvation-induced metabolic acidosis in a non-diabetic adolescent with anorexia nervosa. In this case, the intricacy of an eating disorder with a bipolar disorder, a neurological pediatric condition and a complex family setting could be aggravating factors that enabled and prolonged both the aphagia and the secondary acute metabolic episode. Metabolic acidosis requires urgent medical intervention because of the short-term risk of death. The difficulty of the management in this specific case lies in the multiplicity of the factors involved, which requires a specialized inpatient approach provided by a transdisciplinary medical team taking into account somatic, nutritional, psychiatric, individual and familial aspects.

\section{References}

1. Campbell K, Peebles R. Eating disorders in children and adolescents: state of the art review. Pediatrics. 2014;134(3):582-592. doi: 10.1542/ peds.2014-0194.

2. Guidelines of the French Authority for Health (HAS)
3. Chalela JA, Lopez JI. Medical management of hunger strikers. Nutr Clin Pract. 2013;28(1):128-135.

4. Gétaz L, Rieder JP, Nyffenegger L, Eytan A, Gaspoz JM, Wolff H. Hunger strike among detainees: guidance for good medical practice. Swiss Med Wkly. 2012;142:w13675. doi: 10.4414/smw.2012.13675.

5. Frise CJ, Mackillop L, Joash K, Williamson C. Starvation ketoacidosis in pregnancy. Eur J Obstet Gynecol Reprod Biol. 2013;167(1):1-7. doi: 10.1016/j.ejogrb.2012.10.005

6. Burbos N, Shiner AM, Morris E. Severe metabolic acidosis as a consequence of acute starvation in pregnancy. Arch Gynecol Obstet. 2009;279(3):399-400. doi: 10.1007/s00404-008-0715-3.

7. Dwyer JB, Tamama K. Ketoacidosis and trace amounts of isopropanol in a chronic alcoholic patient. Clin Chim Acta. 2013;16:415:245-249. doi: 10.1016/j.cca.2012.10.057.

8. D, Rehm M, Da Si lva L,Christ F, Finsterer U. Severe metabolic acidosis resulting from a dislocated gastric band. Obes Surg. 2004;14(4):555558.

9. Chalasani S, Fischer J. South Beach Diet associated ketoacidosis: a case report. J Med Case Rep. 2008;2:45. doi: 10.1186/1752-1947-2-45

10. Shah $\mathrm{P}$, Isley WL. Ketoacidosis during a low-carbohydrate diet. N Engl J Med. 2006;354(1):97-98. DOI: 10.1056/NEJMc052709

11. Mittal M, Khan S. Starvation causes acute psychosis due to anterior thalamic infarction. South Med J. 2010;103(7):701-703.

12. Levraut J, Grimaud D. Treatment of metabolic acidosis. Curr Opin Crit Care. 2003;9(4):260-265

13. McElroy SL, Kotwal R, Keck PE Jr, Akiskal HS. Comorbidity of bipolar and eating disorders: distinct or related disorders with shared dysregulations? J Affect Disord. 2005;86(2-3):107-127.

14. McElroy SL, Frye MA, Hellemann G, Altshuler L, Leverich GS, Suppes 